\title{
Killing Orbits and Isotropy in General Relativity
}

\section{Graham Hall*}

Institute of Mathematics, University of Aberdeen, Aberdeen AB24 3UE, Scotland, UK

This brief note is intended to show how a global and rigorous approach to certain problems regarding symmetry in general relativity theory may be given using the theory of Killing orbits and isotropy. Examples from cosmology and plane wave theory are chosen to illustrate the method. More details on these examples may be found in [1] and on the general techniques used in $[2,3]$.

Let $(M, g)$ be a space-time and let $K(M)$ denote the Lie algebra (under the usual Lie bracket of vector fields) of all global, Killing vector fields on $M$. Thus $X \in K(M) \Leftrightarrow L_{X} g=0$ where $\mathcal{L}$ denotes the Lie derivative. In any coordinate domain of $M$ any $X \in K(M)$ satisfies $X_{a ; b}=F_{a b}=-F_{b a}$ where a semi-colon denotes a covariant derivative and $F$ is the Killing bivector associated with $X$. If $T M$ denotes the tangent space to $M$ at $m$ let $f: K(M) \rightarrow T_{m} M$ denote the linear map $X \rightarrow X(m)$ with range space $D_{m} \equiv\{X(m): X \in K(M)\}$ and kernel $I_{m} \equiv\{K(M): X(m)=0\}$ Thus $\operatorname{dim} K(M)=\operatorname{dim} D_{m}+\operatorname{dim} I_{m} . I_{m}$ is a subalgebra of $K(M)$ called the isotropy subalgebra and the map $D: m \rightarrow D_{m}$ is a generalised distribution on $M$ (that is, it is not necessarily a Frobenius distribution since $\operatorname{dim} D_{m}$ may not be constant on $M)$. Since any $X \in K(M)$ is uniquely determined by the values $X(m)$ and $F(m)$ at any $m \in M$, it follows that $\operatorname{dim} K(M) \leq 10$ and that any $X \in I_{m}$ is uniquely determined by its associated $F(m)$. It may then be checked that $I_{m}$ is Lie isomorphic to $\{F(m): F$ the Killing bivector of some $\left.X \in I_{m}\right\}$ under the map $X \rightarrow F(m)$, the latter subalgebra under the Lie product of matrix commutation. It then follows that $I_{m}$ is Lie isomorphic to a subalgebra of the Lorentz algebra $\mathrm{L}$ of the Lorentz group and a classification of such subalgebras is well known [4].

Each $X \in K(M)$ gives rise to a collection of local flow diffeomorphisms $d_{t}$ which are local diffeomorphisms of $M$ and which essentially move each point of some open subset of $M$ a parameter distance $t$, for $t$ in some open interval of $R$, along the integral curves of $X$. For $m \in M$ the subset $O_{m}$ of points of $M$ to which $m$ may be mapped under successive applications of the permissible $d_{t}$ arising from $K(M)$ is called the orbit (through $m$ ) associated with $K(M)$. Such subsets are connected submanifolds of $M$ and are integral manifolds of the distribution $D$, The nature of an orbit (null, spacelike or timelike) is the same at all its points. Then $\operatorname{dim} D_{m}=\operatorname{dim} O_{m}$ and $\operatorname{so} \operatorname{dim} K(M)=\operatorname{dim} O_{m}+\operatorname{dim} I_{m}$ for each $m \in M$. If $X \in K^{m}(M)$ and $X(m)=0, m$ is a zero of $X$ and also a fixed point of any $d_{t}$ arising from $X, d_{t}(m)=m$. Then the linear (pushforward) map $d_{t}^{*}$ "reflects" the symmetry from $X$ in the tangent space $T_{m} M$ and the other tensor spaces at $\mathrm{m}$.

The dimensions of $K(M)$ and the orbits associated with $K(M)$ can be related in the following way. First an orbit $O$ of $K(M)$ is called dimensionally stable if whenever $m \in O$ there exists a neighbourhood $U$ of $m$ such that the dimension of any orbit through any point of $U$ has the same dimension as $O$. The following can then be shown. (a) If $K(M)$ admits a 3-dimensional null orbit, $3 \leq \operatorname{dim} K(M) \leq 7$, whilst if $K(M)$ admits any dimensionally stable, 3-dimensional null orbit or any 3-dimensional, non-null orbit (the latter being necessarily dimensionally stable), $3 \leq \operatorname{dim} K(M) \leq 6$. (b) If $K(M)$ admits a 2-dimensional, null (respectively a 2 -dimensional non-null) orbit, $2 \leq$ $\operatorname{dim} K(M) \leq 5$, (respectively, $2 \leq \operatorname{dim} K(M) \leq 4$ ), whilst if $K(M)$ admits any 2-dimensional, dimensionally stable orbit, $2 \leq \operatorname{dim} K(M) \leq 3$. (c) If $K(M)$ admits a 1-dimensional, null (respectively a 1-dimensional non-null) orbit, $1 \leq \operatorname{dim} K(M) \leq 5$, (respectively, $1 \operatorname{dim} K(M) \leq 4$ ), whilst if $K(M)$ admits any 1-dimensional, dimensionally stable orbit, $\operatorname{dim} K(M)=1$. These results, together with the above theory, may be used to give a global approach to some problems with high symmetry and from which the usual results, often obtained by somewhat ad hoc methods, may be derived. Two examples drawn from cosmology and plane wave theory are used to clarify the ideas.

First, consider cosmological models. The concept of isotropy ("all directions are equivalent") only makes sense if one takes it to mean that all incoming null directions (of photons from observations) are, in some sense, "equivalent". So call a space-time cosmological if, for each $m \in M$, the collection of linear maps $\mathrm{d}^{*}$ arising from members of $I_{m}$ act transitively on the set of null directions at $m$. This imposes a restriction on the Lie algebra $I$ and, following a check on the collection of possible subalgebras of $L$, it turns out that $I_{m}$ can only be Lie isomorphic to $o$ (3) (subalgebra $R_{13}$ in [4]) or $L$ itself (subalgebra $R_{15}$ ). However, using the theory of the previous paragraphs, it can be shown that one necessarily has the global results that either $I_{m} \approx o(3)$ at every $m \in M$ or $I \approx L$ at every $m \in M$. Using the results on the relationship between $\operatorname{dim} K(M)$ and orbit dimension, one then finds that any orbit of $K(M)$ has dimension 3 or 4 and $(M, g)$ consists of either a single 4-dimensional orbit or each of its orbits is 3-dimensional and spacelike. All orbits are dimensionally stable. Further one easily shows that either (i) $I_{m}$ is isomorphic to $L$ for each $m \in M$, in which case, $(M$, $g$ ) has constant curvature and $\operatorname{dim} K(M)=10$ with $K(M)$ having a single 4-dimensional orbit, or, (ii) $I_{m}$ is isomorphic to $s o(3)$ at each $m \in M$ and $K(M)$ has a single 4-dimensional orbit. In this case $\operatorname{dim} K(\mathrm{M})=7$, or, (iii) $I_{m}$ is isomorphic to $o(3)$ for each $m \in M$ and each orbit of $K(M)$ is 3 -dimensional and spacelike. In this case $\operatorname{dim} K(M)=6$. If case $(i)$ holds, $(M, g)$ is of constant curvature and hence is locally de Sitter, anti-de Sitter, or Minkowski space whilst if case (ii) holds, $(M, g)$ is either of constant curvature or locally of the Einstein static type. If case (iii) holds, $(M, g)$ admits an open dense subset $V$ such that each $m \in \mathrm{V}$ admits a neighbourhood whose restricted metric is either of constant curvature or of the (generic) FRWL type. [It is noted that there is no contradiction if $(M, g)$ is of constant curvature and $\operatorname{dim} K(M)<10$; however the local Killing algebra will be 10-dimensional, (see e.g. p 337.) The smoothness of the relevant geometrical objects on $M$ follows from the smoothness of $\mathrm{g}$ and the members of $K(M)$. One thus achieves a description of all possible models satisfying the above cosmological condition. When $I_{m} \approx 0(3)$ a unique timelike direction at $\mathrm{m}$ is determined and which is preserved by each $\mathrm{dt}^{*}$ (the "cosmological observer").

The assumption that $(M, g)$ is cosmological also forces "homogeneity" onto $(M, g)$ in the sense that either there exists a

*Corresponding author: Graham Hall, Institute of Mathematics, University of Aberdeen, Aberdeen AB24 3UE, Scotland, UK, E-mail: g.hall@abdn.ac.uk

Received April 23, 2013; Accepted April 24, 2013; Published April 26, 2013

Citation: Hall G (2013) Killing Orbits and Isotropy in General Relativity. J Appl Computat Math 2: e130. doi:10.4172/2168-9679.1000e130

Copyright: @ 2013 Hall G. This is an open-access article distributed under the terms of the Creative Commons Attribution License, which permits unrestricted use, distribution, and reproduction in any medium, provided the original author and source are credited. 
4-dimensional orbit (transitivity) or the orbits are 3-dimensional and give a local cosmic time function (and in (ii), although the Killing orbit is 4-dimensional, a 6-dimensional subalgebra of $K(M)$ may be shown to exist whose 3-dimensional integral manifolds give a local cosmic time function).

One may also apply this method to a study of plane wave symmetry. Let $(M, g)$ be a space-time and let $l$ be a null direction at $m \in M$. A wave surface of $l$ at $m$ is a spacelike, 2-dimensional subspace of $T_{m} M$ orthogonal to $l$. If $l, n, x, y$ is a null tetrad at $m$, the wave surfaces of $l$ at $m$ can be represented in a one-to-one way (by the pair $(a, b)$ with $a, b \in R$ ) by those subspaces spanned by vectors of the form $x+a l$ and $y+b l$. Each unit timelike vector $u$ at $m$ determines a unique wave surface of $l$ at $m$ which is orthogonal to $u$ (and $l$ ) and is then the instantaneous wave surface of $l$ at $m$ for the observer with 4 -velocity $u$ at $m$. Suppose one attempts to propose a definition of a plane wave $(M$, $g$ ) by insisting that the Killing algebra $K(M)$ of $(M, g)$ is such that for each $m \in M$, there is a unique null direction $l \in T_{m} M$ such that the $d_{t}^{*}$ arising from members of $I_{m}$ are transitive on the set of wave surfaces of $l$ at $m$. One can again check the subalgebras of $L$ for candidates for $I_{m}$. The situation is a little more complicated now and it turns out that five subalgebras of $L$ are possible and which have dimension 2 or 3 . However, in all cases, the maps $d_{t}^{*}$ fix the direction of $l$ at $m$ and it can be shown that this is the only direction that they fix. Then, as in the cosmological case, one can achieve a global description of all such models. In fact, if one further restricts by the assumptions that all orbits of $K(M)$ are dimensionally stable and that no non-empty open subsets of $M$ of constant curvature exist then one gets the results that either $I$ is 2 -dimensional at each with $\operatorname{dim} K(M)=5$ (respectively, $\operatorname{dim} K(M)=6$ ) and all orbits are 3-dimensional and null (respectively, there exists a single 4-dimensional orbit), or $I_{m}$ is 3-dimensional at each $m \in M$ with $\operatorname{dim} K(M)=6$ (respectively, $\operatorname{dim} K(M)=7$ ) and all orbits are 3-dimensional and null (respectively, there exists a single 4-dimensional orbit). These solutions can then be shown to give the usual (Petrov type $\mathrm{N}$ or $\mathrm{O}$ ) plane wave metrics including, of course, the homogeneous ones and the one with non-zero cosmological constant given in $[5,6]$.

\section{References}

1. Hall GS (2013) Symmetries, Orbits and Isotropies in General Relativity Theory. Preprint, University of Aberdeen.

2. Hall GS (2004) Symmetries and Curvature Structure in General Relativity World Scientific.

3. Hall GS (2003) Class Quant Grav 20: 4067.

4. Schell J F (1961) Classification of Four-Dimensional Riemannian Spaces. J Math Phys 2: 202.

5. Defrise $L$ (1969) Groupes d'isotropie et groupes de stabilite conforme dans les espaces Lorentziens. Thesis, Universite Libre de Bruxelles.

6. Stephani H, Kramer D, Mac Callum MAH, Hoenselaers C and Herlt E (2003) Exact Solutions of Einstein's field Equations (Second Edition) Cambridge. 